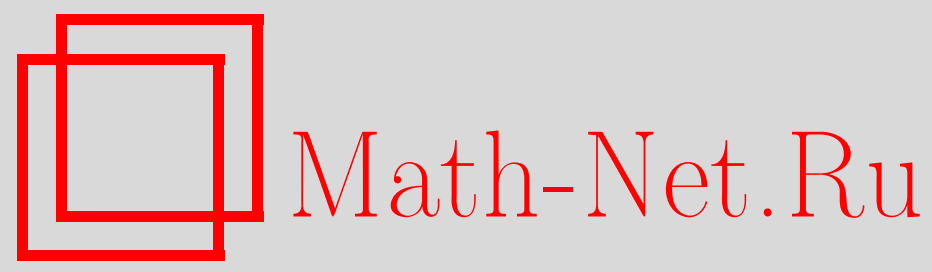

А. В. Абанин, Г. А. Семенова, Об одном обобщении ряда Лорана, Матем. заметки, 2000, том 68, выпуск 1, 3-12

DOI: https://doi.org/10.4213/mzm914

Использование Общероссийского математического портала Math-Net.Ru подразумевает, что вы прочитали и согласны с пользовательским соглашением http://www.mathnet.ru/rus/agreement

Параметры загрузки:

IP: 54.172 .240 .79

26 апреля 2023 г., 13:22:40

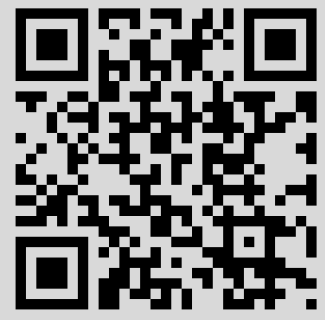




\title{
ОБ ОДНОМ ОБОБЩЕНИИ РЯДА ЛОРАНА
}

\author{
А. В. Абанин, Г. А. Семенова
}

В статье исследуется вопрос о том, при каких условиях на область $G$ в расширенной комплексной плоскости $\overline{\mathbb{C}}$ и последовательность точек $\Lambda=\left\{\lambda_{k}\right\}$ в дополнении $G^{c}=\overline{\mathbb{C}} \backslash G$ к области $G$ любая функция $f(z)$, голоморфная в $G$ и равная нулю в бесконечно удаленной точке $z=\infty$, если $\infty \in G$, допускает (возможно неединственное) представление в виде равномерно и абсолютно сходящегося внутри $G$ ряда

$$
f(z)=\sum_{k=1}^{\infty} \sum_{j=1}^{\infty} \frac{a_{k, j}}{\left(z-\lambda_{k}\right)^{j}}, \quad z \in G .
$$

Если $\bar{G} \subset \mathbb{C}$ или $G^{c} \subset \mathbb{C}$, то такое разложение имеет место тогда и только тогда, когда для любого $\varepsilon>0$ система кругов $\left\{K\left(\lambda_{k}, \operatorname{dist}\left(\lambda_{k}, \partial G\right)+\varepsilon\right)\right\}_{\lambda_{k} \in \Lambda}$ покрьвает $\partial G$.

Библиограбоия: 15 названий.

1. Введение. Возможность представления аналитических функций рядами простейших дробей вида

$$
\sum_{k=1}^{\infty} \frac{a_{k}}{z-\lambda_{k}}, \quad a_{k}, \lambda_{k} \in \mathbb{C}, \quad \sum_{k=1}^{\infty}\left|a_{k}\right|<\infty,
$$

а также различные задачи, связанные с такими представлениями, исследовались многими авторами (см., например, [1]-[6]). Известно [5], что ни для одной области $G$ расширенной комплексной плоскости $\widehat{\mathbb{C}}$ не существует такой последовательности $\left\{\lambda_{k}\right\}$, чтобы каждую функцию из $H_{0}(G)$ можно было представить в виде суммы ряда (1) (здесь и далее $H_{0}(G)$ - пространство всех функций, аналитических в $G$ и исчезаюших в бесконечно удаленной точке, если она принадлежит $G)$. Более того, последнее невозможно также ни для одной системы дробей вида $\left\{\left(z-\lambda_{k}\right)^{-j}: j=1,2, \ldots, j_{k} ; k \in \mathbb{N}\right\}$, если $\sup \left\{j_{k}: k \in \mathbb{N}\right\}<+\infty$. С другой стороны, в [7], [8] установлен следующий результат.

ТЕОрема 1. Пусть $F$ - континуум в комплексной плоскости $\mathbb{C}, G$ - открытое дополнение $F$ до расширенной комплексной плоскости $\widehat{\mathbb{C}},\left\{\lambda_{k}\right\}-$ плотная в $F$ последовательность точек. Если $f(z)$ аналитична в $G$ и $f(\infty)=0$, то существуют функиии $f_{1}(z), \ldots, f_{k}(z), \ldots$ такие, что $f(z)=\sum_{k=1}^{\infty} f_{k}(z)$, причем ряд сходится

Работа выполнена при поддержке Российского фонда фундаментальных исследований, грант № 99-01-00178. 
равномерно на каждом замкнутом в $G$ множестве, а функиия $f_{k}(z)$ регулярна всюду, кроме $\lambda_{k}(k=1,2, \ldots)$.

В процессе доказательства в [8] установлено фактически, что

$$
f(z)=\sum_{k=1}^{\infty} \sum_{j=1}^{\infty} \frac{a_{k, j}}{\left(z-\lambda_{k}\right)^{j}}, \quad z \in G
$$

причем ряд сходится абсолютно в пространстве $H_{0}(G)$ при наделении его естественной топологией пространства $\Phi$ реше. В случае, когда $F$ состоит из одной точки $a \in \mathbb{C}$, имеем разложение произвольной функции, аналитической в $\widehat{\mathbb{C}} \backslash\{a\}$ и исчезающей в бесконечности, в ряд Лорана.

В настоящей работе устанавливаются связьвающие между собой область $G$ расширенной комплексной плоскости $\widehat{\mathbb{C}}$ и распределение точек $\left\{\lambda_{k}\right\}$ в ее дополнении до $\mathbb{C}$ критерии того, что для каждой функции $f$ из $H_{0}(G)$ справедливо абсолютно сходящееся в $H_{0}(G)$ разложение типа $(2)$.

2. Формулировка основного результата. Пусть $G$ - область в $\widehat{\mathbb{C}}$, отличная от всей расширенной плоскости $\widehat{\mathbb{C}} ;\left\{G_{n}\right\}_{n=1}^{\infty}-$ последовательность областей в $\widehat{\mathbb{C}}$, исчерпывающая $G$ изнутри (т.е. $\bigcup_{n=1}^{\infty} G_{n}=G$ и $\bar{G}_{n} \subset G_{n+1}, n \in \mathbb{N}$, где замыкание берется в $\left.\widehat{\mathbb{C}}\right)$. Символом $H_{c, 0}\left(G_{n}\right)$ обозначим пространство всех функций, аналитических в $G_{n}$, непрерьвных в $\bar{G}_{n}$ и исчезающих в бесконечно удаленной точке, если она принадлежит $G_{n}$. $H_{c, 0}\left(G_{n}\right)$ наделяется топологией, задаваемой нормой $\|f\|_{n}:=\max \left\{|f(z)|: z \in \partial G_{n}\right\}$, $n \in \mathbb{N}$. Поскольку $H_{0}(G)$ плотно в каждом $H_{c, 0}\left(G_{n}\right)$, то $H_{0}(G)$ - так называемьй (см. [9]) приведенньй проективньй предел банаховых пространств $H_{c, 0}\left(G_{n}\right)$ относительно операций сужения. Как известно, введенная топология не зависит от выбора исчерпывающей $G$ последовательности областей $\left\{G_{n}\right\}_{n=1}^{\infty}$ и может быть задана набором преднорм $p_{T}(f):=\max \{|f(z)|: z \in T\}$, где $T-$ произвольньй компакт в $G$. В силу этого обстоятельства мы имеем возможность проводить рассуждения для какой-либо фиксированной последовательности областей $\left\{G_{n}\right\}_{n=1}^{\infty}$, исчерпывающей $G$ изнутри, подчиняя ее, если потребуется, дополнительным ограничениям.

Для произвольной точки $\lambda \in \mathbb{C}$ и множества $D \subset \widehat{\mathbb{C}}$ обозначим через $\rho(\lambda, D)$ евклидово расстояние от $\lambda$ до $D \backslash\{\infty\}$, а через $K(\lambda, r)$, где $0<r<\infty,-$ открытьй круг с центром в $\lambda$ радиуса $r$. Далее, символом $M(\lambda, D)$ будем обозначать множество точек из $D$, “реализующих" расстояние от $\lambda$ до $D$, т. е. $M(\lambda, D):=\{z \in D: \rho(\lambda, D)=|z-\lambda|\}$. Если $\Lambda \subset \mathbb{C}$, то полагаем $M(\Lambda, D)=\bigcup_{\lambda \in \Lambda} M(\lambda, D)$.

Основной результат работы составляет

Теорема 2. Пусть $G$ - конечносвязная область в $\widehat{\mathbb{C}}$, отличная от $\widehat{\mathbb{C}}$ и от $\mathbb{C}$, $\Lambda=\left\{\lambda_{k}\right\}-$ не более чем счетное мноэсество точек из $\mathbb{C} \backslash G$. Следуюшие условия әквивалентны:

(i) каждую функиию $f(z)$ из $H_{0}(G)$ можсно представить в виде суммы ряда (2), абсолютно сходящегося в $H_{0}(G)$, (m.e. $\sum_{k} \sum_{j=1}^{\infty}\left|a_{k, j}\right|\left(\rho\left(\lambda_{k}, T\right)\right)^{-j}$ сходится для любого компакта $T$ из $G)$;

(ii) для любого компакта $S \subset G$ найдется такой компакт $T \subset G$, что система кругов $\left\{K\left(\lambda_{k}, \rho\left(\lambda_{k}, S\right)\right)\right\}_{\lambda_{k} \in \Lambda}$ покрывает $G \backslash T$. 
В случае, когда $G$ ограничена или содержит бесконечно удаленную точку, (i) и (iі) равносильны такому условию:

(iii) для любого $\varepsilon>0$ система кругов $\left\{K\left(\lambda_{k}, \rho\left(\lambda_{k}, \partial G\right)+\varepsilon\right)\right\}_{\lambda_{k} \in \Lambda}$ покрыввает $\partial G$.

А если дополнительно ко всему известно, что $\Lambda$ ограничено, то еще и такому условию:

(iv) $M(\bar{\Lambda}, \partial G)=\partial G$, əде $\bar{\Lambda}-$ замыкание $\Lambda$ в $\mathbb{C}$.

ЗАмЕчАнИЕ. Ниже будет показано, что все дополнительные требования (конечносвязности, ограниченности $G$ или ее дополнения до $\widehat{\mathbb{C}}$, а также ограниченности $\Lambda$ ) существенны для справедливости соответствующих эквивалентностей.

3. Функциональный критерий. Доказательство теоремы 2 базируется на применении теории абсолютно представляющих систем [10], которая позволяет свести задачу о представлениях вида (2) к двойственной задаче аналитического продолжения с равномерными оценками. Для формулировки и доказательства соответствующего критерия потребуются некоторые вспомогательные обозначения, определения и результаты.

Пусть $G$ - область в $\widehat{\mathbb{C}}, G^{c}$ - ее дополнение до $\widehat{\mathbb{C}}, H_{0}\left(G^{c}\right)$ - пространство ростков аналитических на замкнутом множестве $G^{c}$ функций, исчезающих в бесконечно удаленной точке, если она принадлежит $G^{c}$, наделенное топологией индуктивного предела относительно операций вложения в него банаховых пространств $H_{c, 0}\left(\bar{G}_{n}^{c}\right)$.

Символом $H_{0}^{\prime}(G)$ обозначим сильное сопряженное с $H_{0}(G)$ пространство. Топологически оно представляет собой внутренний индуктивньй предел банаховьх пространств

$$
E_{n}=\left\{\Phi \in H_{0}^{\prime}(G):\|\Phi\|_{n}^{\prime}=\sup \left\{|\Phi(f)|: f \in H_{0}(G),\|f\|_{n} \leqslant 1\right\}<\infty\right\}
$$

где $n \in \mathbb{N}$. Как известно [11] (см. также [12]), преобразование Коши

$$
L: \Phi \in H_{0}^{\prime}(G) \rightarrow \Phi_{z}\left(\frac{1}{z-\lambda}\right)=: \varphi(\lambda), \quad \lambda \in G^{c}
$$

является топологическим изоморфизмом $H_{0}^{\prime}(G)$ на $H_{0}\left(G^{c}\right)$. При этом в силу факторизационной теоремы Гротендика [13, теорема 6.5.1]

$$
\forall n \in \mathbb{N} \quad \exists m \in \mathbb{N} \mid L: E_{n} \rightarrow H_{c, 0}\left(\bar{G}_{m}^{c}\right) \text { и } L^{-1}: H_{c, 0}\left(\bar{G}_{n}^{c}\right) \rightarrow E_{m}
$$

- непрерьвные операторы.

Для системы $F(\Lambda):=\left\{\left(z-\lambda_{k}\right)^{-j}: j \in \mathbb{N}, \lambda_{k} \in \Lambda\right\}$ введем в рассмотрение подпространства в $H_{0}\left(G^{c}\right)$

$$
F_{\Lambda, n}=\left\{\varphi \in H_{0}\left(G^{c}\right):|\varphi|_{\Lambda, n}=\sup _{k} \sup _{j \in \mathbb{N}} \frac{\left|\varphi^{(j-1)}\left(\lambda_{k}\right)\right|}{(j-1) !}\left(\rho\left(\lambda_{k}, \partial G_{n}\right)\right)^{j}<\infty\right\},
$$

и в $H_{0}^{\prime}(G)$

$$
E_{\Lambda, n}:=\left\{\Phi \in H_{0}^{\prime}(G):|\Phi|_{\Lambda, n}^{\prime}:=\sup _{k} \sup _{j \in \mathbb{N}} \frac{\left|\Phi_{z}\left(\left(z-\lambda_{k}\right)^{-j}\right)\right|}{\left\|\left(z-\lambda_{k}\right)^{-j}\right\|_{n}}<\infty\right\},
$$


где $n=1,2, \ldots$

Напомним еще, что система $\left\{x_{m}\right\}_{m=1}^{\infty}$ элементов локально выпуклого пространства $H$ называется абсолютно представляющей в $H$ [10], если каждьй элемент $x$ из $H$ можно представить (не обязательно единственным способом) в виде суммы ряда $x=$ $\sum_{m=1}^{\infty} c_{m} x_{m}\left(c_{m}-\right.$ скаляры $)$, абсолютно сходящегося в $H$. В соответствии с этим определением условие (i) теоремы 2 означает, что $F(\Lambda)$ - абсолютно представляющая в $H_{0}(G)$ система.

ТЕорема 3. Для того чтобы система $F(\Lambda)$ была абсолютно представляющей в $H_{0}(G)$, необходимо и достаточно, чтобы для любого $n \in \mathbb{N}$ нашлись такие $m=m(n) \in \mathbb{N} u A=A(n)>0$, чтобы каждая функиия $\varphi$ из $F_{\Lambda, n}$ обладала аналитическим продолжением в $\bar{G}_{m}^{c}$, непрерывным в $G_{m}^{c}$, и для әтого продолэсения имела место равномерная по ч оченка

$$
\|\varphi\|_{m} \leqslant A|\varphi|_{\Lambda, n}
$$

ДОКАЗАТЕЛЬСТВо. В соответствии с теоремой 3 главы 1 из [10] $F(\Lambda)$ - абсолютно представляющая в $H_{0}(G)$ система в том и только том случае, когда

$$
\forall n \in \mathbb{N} \exists p \in \mathbb{N} \mid E_{\Lambda, n} \hookrightarrow E_{p}
$$

(здесь и далее $\hookrightarrow-$ символ непрерывного вложения). Прямой подсчет показывает, что если $\varphi$ из $H_{0}\left(G^{c}\right)$ и $\Phi$ из $H_{0}^{\prime}(G)$ связаны преобразованием Коши $\varphi=L(\Phi)$, то для любых $\lambda \in \mathbb{C} \backslash G, j \in \mathbb{N}, n \in \mathbb{N}$

$$
\Phi_{z}\left((z-\lambda)^{-j}\right)=\frac{\varphi^{(j-1)}(\lambda)}{(j-1) !} \quad \text { и } \quad\left\|(z-\lambda)^{-j}\right\|_{n}=\left(\rho\left(\lambda, \partial G_{n}\right)\right)^{-j} .
$$

Поэтому $L: E_{\Lambda, n} \rightarrow F_{\Lambda, n}-$ изоморфньй оператор, сохраняющий норму, и, следовательно, (5) можно переписать так:

$$
\forall n \in \mathbb{N} \quad \exists p \in \mathbb{N} \mid L^{-1}\left(F_{\Lambda, n}\right) \hookrightarrow E_{p} .
$$

Применив (3), получим отсюда, что $F(\Lambda)$ тогда и только тогда является абсолютно представляющей в $H_{0}(G)$ системой, когда

$$
\forall n \in \mathbb{N} \exists m \in \mathbb{N} \mid F_{\Lambda, n} \hookrightarrow H_{c, 0}\left(\bar{G}_{m}^{c}\right),
$$

а это эквивалентно тому, что каждая функция $\varphi$ из $F_{\Lambda, n}$ обладает аналитическим продолжением в $\bar{G}_{m}^{c}$, непрерывным в $G_{m}^{c}$, и для этого продолжения справедлива равномерная по $\varphi$ оценка (4).

ЗАмечАниЕ. Теорема 3 имеет место также и для более общих систем, чем $F(\Lambda)$, а именно для $F_{J}(\Lambda):=\left\{\left(z-\lambda_{k}\right)^{-j}: j \in J_{k}, \lambda_{k} \in \Lambda\right\}$, где $J=\left\{J_{k}\right\}$, а $J_{k}-$ произвольные подмножества натуральных чисел. Ясно, что при этом в формулировке следует естественным образом изменить определение $F_{\Lambda, n}$ и $|\varphi|_{\Lambda, n}$, а доказательство остается прежним. Мы ограничились здесь случаем $F(\Lambda)=F_{\{\mathbb{N}\}}(\Lambda)$, поскольку содержательные результаты удалось установить лишь для таких систем. Следует отметить, что это ограничение вызвано также существом дела. Например, если $G$ - внешность замкнутого круга $\{z:|z-\lambda| \leqslant R\}, 0 \leqslant R<\infty, \Lambda$ состоит ровно из одной точки $\lambda \in \mathbb{C}$ (центра круга), то по теореме Лорана система степеней $\left\{(z-\lambda)^{-j}: j \in J\right\}$ является абсолютно представляющей в $H_{0}(G)$ в том и только том случае, когда $J=\mathbb{N}$ (очевидно, в этой ситуации она будет базисом). 
4. Доказательство теоремы 2. Пусть выполнено условие (i). Зафиксируем произвольный компакт $S \subset G$ и найдем такое $n \in \mathbb{N}$, чтобы $S \subset \bar{G}_{n}$. При каждом $z$ из $G \backslash \bigcup_{k} K\left(\lambda_{k}, \rho\left(\lambda_{k}, S\right)\right)$ рассмотрим функцию $\varphi_{z}(\lambda)=(z-\lambda)^{-1} \in H_{0}\left(G^{c}\right)$. Так как $\left|z-\lambda_{k}\right| \geqslant \rho\left(\lambda_{k}, S\right) \geqslant \rho\left(\lambda_{k}, \partial G_{n}\right)$, то

$$
\sup _{k} \sup _{j \in \mathbb{N}} \frac{\left|\varphi_{z}^{(j-1)}\left(\lambda_{k}\right)\right|}{(j-1) !}\left(\rho\left(\lambda_{k}, \partial G_{n}\right)\right)^{j}=\sup _{k} \sup _{j \in \mathbb{N}}\left(\frac{\rho\left(\lambda_{k}, \partial G_{n}\right)}{\left|z-\lambda_{k}\right|}\right)^{j} \leqslant 1 .
$$

Тогда в соответствии с теоремой 3 каждая функция $\varphi_{z}(\lambda)$ обладает аналитическим продолжением в некоторое $\bar{G}_{m}^{c}$, являющимся к тому же непрерывным в $G_{m}^{c}$. А для этого необходимо и достаточно, чтобы рассматриваемые $z$ лежали в $G_{m}$. Таким образом, $G \backslash \bigcup_{k} K\left(\lambda_{k}, \rho\left(\lambda_{k}, \partial G_{n}\right)\right)$ вложено в $G_{m}$ и, тем более, $G \backslash \bar{G}_{m}$ вложено в $\bigcup_{k} K\left(\lambda_{k}, \rho\left(\lambda_{k}, S\right)\right)$. Остается положить $T=\bar{G}_{m}$ и условие (iі) вьполнено.

Пусть имеет место (ii). По условию $G$ имеет конечную связность, которую мы обозначим через $s$. Тогда в соответствии с $[14$, c. 21 , следствие] можно считать, что каждая область $G_{n}$ имеет ту же связность и ограничена попарно непересекающимися контурами $\Gamma_{n, 1}, \ldots, \Gamma_{n, s}$, которые ограничивают также соответствующие компоненты связности $Q_{n, 1}, \ldots, Q_{n, s}$ замкнутого множества $G_{n}^{c}$, упорядоченные в соответствии с правилом $Q_{n+1, r} \subset Q_{n, r}(r=1, \ldots, s ; n=1,2, \ldots)$.

Зафиксируем $n \in \mathbb{N}$ и рассмотрим компакт $\partial G_{n}$. По условию (iі) найдется компакт $T$ из $G$ такой, что $\left\{K\left(\lambda_{k}, \rho\left(\lambda_{k}, \partial G_{n}\right)\right)\right\}_{\lambda_{k} \in \Lambda}$ покрьвает $G \backslash T$. Выберем $m \in \mathbb{N}$ так, чтобы $T \subset G_{m}$. Тогда эта система кругов покрьвает также $G \backslash G_{m}$. Поскольку $\partial G_{m} \subset G$ и $\partial G_{m} \cap G_{m}=\varnothing$, то

$$
\bigcup_{k} K\left(\lambda_{k}, \rho\left(\lambda_{k}, \partial G_{n}\right)\right) \supset \partial G_{m}
$$

Теперь рассмотрим произвольную функцию $\varphi \in F_{\Lambda, n}$. При любом фиксированном $k$ и произвольном $z \in \mathbb{C}$

$$
\frac{\left|\varphi^{(j-1)}\left(\lambda_{k}\right)\right|}{(j-1) !}\left|z-\lambda_{k}\right|^{j-1} \leqslant \frac{|\varphi|_{\Lambda, n}}{\rho\left(\lambda_{k}, \partial G_{n}\right)}\left(\frac{\left|z-\lambda_{k}\right|}{\rho\left(\lambda_{k}, \partial G_{n}\right)}\right)^{j-1}, \quad j \in \mathbb{N} .
$$

Отсюда следует, что ряд Тейлора функции $\varphi$ с центром в точке $\lambda_{k}$ сходится по крайней мере в круге $K\left(\lambda_{k}, \rho\left(\lambda_{k}, \partial G_{n}\right)\right)$. Обозначим через $r(k)$ номер (по $r$ ) той компоненты $Q_{n, r}$, в которую попало $\lambda_{k}$. Этот номер, очевидно, не зависит от $n$. Далее, поскольку $\varphi \in H_{0}\left(G^{c}\right)$, имеется такое $p=p(\varphi) \in \mathbb{N}$, что $\varphi \in H_{c, 0}\left(G_{p}^{c}\right)$. Пусть $\varphi_{r}-$ сужение $\varphi$ на $Q_{p, r}$, являющееся аналитической внутри $Q_{p, r}$ и непрерьвной вплоть до $\Gamma_{p, r}$ функцией $(r=1, \ldots, s)$. Тогда, так как круг $K\left(\lambda_{k}, \rho\left(\lambda_{k}, \partial G_{n}\right)\right)$ попадает только в область int $Q_{n, r(k)}$ и так как (int $\left.Q_{p, r}\right) \cup\left(\bigcup_{k, r(k)=r} K\left(\lambda_{k}, \rho\left(\lambda_{k}, \partial G_{n}\right)\right)\right)$ - область, которую мы обозначим символом $D_{p, r, n}$, то функция

$$
\tilde{\varphi}_{r}(z)= \begin{cases}\varphi_{r}(z), & z \in Q_{p, r}, \\ \sum_{j=1}^{\infty} \frac{\varphi_{r}^{(j-1)}\left(\lambda_{k}\right)}{(j-1) !}\left(z-\lambda_{k}\right)^{j-1}, & z \in K\left(\lambda_{k}, \rho\left(\lambda_{k}, \partial G_{n}\right)\right)\end{cases}
$$


где $k$ пробегает множество $\{k: r(k)=r\}$, определена корректно и является аналитическим продолжением $\varphi_{r}$ в область $D_{p, r, n}$. В соответствии с (6) $D_{p, r, n}$ содержит контур $\Gamma_{m, r}$ при некотором $m=m(n)$. Тогда функция

$$
\psi_{r}(z):= \begin{cases}\frac{1}{2 \pi i} \int_{\Gamma_{m, r}} \frac{\widetilde{\varphi}_{r}(t)}{t-z} d t, & z \in \operatorname{int} Q_{m, r} \\ \widetilde{\varphi}_{r}(z), & z \in \Gamma_{m, r}\end{cases}
$$

где обход $\Gamma_{m, r}$ в интеграле осуществляется в положительном относительно $Q_{m, r}$ направлении, будет аналитическим продолжением $\varphi_{r}$ в область int $Q_{m, r}$, непрерьвньп вплоть до $\Gamma_{m, r}$, а $\psi(z):=\psi_{r}(z), z \in Q_{m, r}, r=1, \ldots, s,-$ аналитическим продолжением $\varphi$ в $\bar{G}_{m}^{c}$, непрерьвным в $G_{m}^{c}$.

Выделим конечное подпокрытие: $\bigcup_{k=1}^{l} K\left(\lambda_{k}, \rho\left(\lambda_{k}, \partial G_{n}\right)\right) \supset \partial G_{m}$ (это возможно, так как $\partial G_{m}-$ компакт в $\left.\mathbb{C}\right)$. Функция

$$
\gamma(t):=\min \left\{\left|t-\lambda_{k}\right| / \rho\left(\lambda_{k}, \partial G_{n}\right): 1 \leqslant k \leqslant l\right\}
$$

непрерьвна в $\mathbb{C}$ и принимает на $\partial G_{m}$ значения, строго меньшие 1 . Тогда

$$
q_{n}:=\max \left\{\gamma(t): t \in \partial G_{m}\right\}<1
$$

и, использовав $(7)$, получим для любой функции $\varphi$ из $F_{\Lambda, n}$

$$
\|\varphi\|_{m} \leqslant|\varphi|_{\Lambda, n}\left(\min _{1 \leqslant k \leqslant l} \rho\left(\lambda_{k}, \partial G_{n}\right)\right)^{-1} \sum_{j=1}^{\infty} q_{n}^{j-1}=A|\varphi|_{\Lambda, n}
$$

где $A=\left(1-q_{n}\right)^{-1}\left(\min _{1 \leqslant k \leqslant l} \rho\left(\lambda_{k}, \partial G_{n}\right)\right)^{-1}$ зависит только от $n$. По теореме 3 отсюда следует, что $F(\Lambda)$ - абсолютно представляющая в $H_{0}(G)$ система, т. е. вьполнено (i). Таким образом, (ii) $\Longleftrightarrow(\mathrm{i})$, и, значит, первые два условия теоремы равносильны между собой.

Предположим, что $G$ ограничена или содержит бесконечно удаленную точку. Ясно, что это предположение эквивалентно ограниченности $\partial G$. Покажем, что (ii) $\Longrightarrow$ (iii).

Допустим, что выполнено (ii). Зафиксируем произвольное $\varepsilon>0$. Так как $\partial G-$ компакт в $\mathbb{C}$, имеется компакт $S$ из $G$, для которого

$$
\rho(z, S)<\frac{\varepsilon}{2} \forall z \in \partial G
$$

Пусть $\xi=\xi(k) \in \partial G$ таково, что $\left|\lambda_{k}-\xi\right|=\rho\left(\lambda_{k}, \partial G\right)$. В силу (8) имеется $\eta=\eta(k) \in S$ $\mathrm{c}|\xi-\eta|<\varepsilon / 2$. Отсюда $\left|\lambda_{k}-\eta\right|<\rho\left(\lambda_{k}, \partial G\right)+\varepsilon / 2$ и, следовательно, $\rho\left(\lambda_{k}, S\right)<\rho\left(\lambda_{k}, \partial G\right)$ $+\varepsilon / 2$. То есть, для любого $\lambda_{k}$ из $\Lambda$ имеет место вложение

$$
K\left(\lambda_{k}, \rho\left(\lambda_{k}, S\right)\right) \subset K\left(\lambda_{k}, \rho\left(\lambda_{k}, \partial G\right)+\frac{\varepsilon}{2}\right) .
$$

По условию (ii) для рассматриваемого компакта $S$ найдется такой компакт $T$ из $G$, что система кругов $\left\{K\left(\lambda_{k}, \rho\left(\lambda_{k}, S\right)\right): \lambda_{k} \in \Lambda\right\}$ покрьвает $G \backslash T$ (можно считать, что $\left.T \supset S\right)$. Тем более этим свойством обладает $\left\{K\left(\lambda_{k}, \rho\left(\lambda_{k}, \partial G\right)+\varepsilon / 2\right): \lambda_{k} \in \Lambda\right\}$. Отсюда, очевидно, следует справедливость (iii). 
Пусть, обратно, имеет место (iіi). Возьмем любой компакт $S \subset G$ и обозначим $\rho(S, \partial G)=\delta$. Из компактности $\partial G$ в $\mathbb{C}$ следует, что существует компакт $T, S \subset T \subset G$, для которого

$$
\rho(z, \partial G) \leqslant \frac{\delta}{2} \quad \forall z \in G \backslash T .
$$

По условию (iii) $\left\{K\left(\lambda_{k}, \rho\left(\lambda_{k}, \partial G\right)+\delta / 2\right): \lambda_{k} \in \Lambda\right\}$ покрывает $\partial G$. Тогда из (9) заключаем, что $\left\{K\left(\lambda_{k}, \rho\left(\lambda_{k}, \partial G\right)+\delta\right): \lambda_{k} \in \Lambda\right\}$ покрывает $G \backslash T$. Оценим теперь $\rho\left(\lambda_{k}, S\right)$. Пусть $\xi \in S$ такова, что $\left|\lambda_{k}-\xi\right|=\rho\left(\lambda_{k}, S\right)$. Так как $\xi \in G$, а $\lambda_{k} \in \mathbb{C} \backslash G$, существует точка $\eta \in \partial G$, лежащая на отрезке, соединяющем $\xi$ и $\lambda_{k}$. Отсюда $\left|\lambda_{k}-\xi\right|=\left|\lambda_{k}-\eta\right|+|\eta-\xi| \geqslant$ $\rho\left(\lambda_{k}, \partial G\right)+\delta$ и, значит, $\rho\left(\lambda_{k}, S\right) \geqslant \rho\left(\lambda_{k}, \partial G\right)+\delta$. Следовательно, для любого $k$ круг $K\left(\lambda_{k}, \rho\left(\lambda_{k}, S\right)\right)$ содержит $K\left(\lambda_{k}, \rho\left(\lambda_{k}, \partial G\right)+\delta\right)$. Тогда в соответствии с предыдущим система кругов $\left\{K\left(\lambda_{k}, \rho\left(\lambda_{k}, S\right)\right): \lambda_{k} \in \Lambda\right\}$ покрьвает $G \backslash T$, т. е. вьполнено (ii). Таким образом, (iii) $\Longrightarrow$ (ii) и, окончательно, (iii) $\Longrightarrow$ (ii) $\Longrightarrow$ (i).

Предположим, наконец, что $\partial G$ и $\Lambda$ ограничены и покажем, что в этом случае (iii) эквивалентно (iv).

Условие (iv) можно переписать в таком виде: $\forall \xi \in \partial G \exists \lambda \in \bar{\Lambda}|\rho(\lambda, \partial G)=| \lambda-\xi \mid$. Тогда его отрицание равносильно тому, что $\exists \xi \in \partial G|\rho(\lambda, \partial G)<| \lambda-\xi \mid, \forall \lambda \in \bar{\Lambda}$. Так как $\bar{\Lambda}$ - компакт в $\mathbb{C}$, для некоторого $\varepsilon_{0}>0 \rho(\lambda, \partial G)+\varepsilon_{0}<|\lambda-\xi| \forall \lambda \in \bar{\Lambda}$. Отсюда следует, что система кружков $\left\{K\left(\lambda_{k}, \rho\left(\lambda_{k}, \partial G\right)+\varepsilon_{0}\right): \lambda_{k} \in \Lambda\right\}$ не содержит $\xi$ и, значит, не покрывает $\partial G$. Таким образом, если не вьполняется (iv), то неверно и (iii). Итак, (iii) $\Longrightarrow$ (iv).

Предположим теперь, что (iv) справедливо. Тогда для каждой точки $\xi \in \partial G$ имеется $\lambda \in \bar{\Lambda} \mathrm{c} \rho(\lambda, \partial G)=|\lambda-\xi|$. Далее, для любого $\varepsilon>0$ найдется $\lambda_{k} \in \Lambda \mathrm{c}\left|\lambda_{k}-\lambda\right|<\varepsilon / 2$. Для этого $\lambda_{k}$ в круге $K\left(\lambda_{k},|\lambda-\xi|-\varepsilon / 2\right)$ нет точек из $\partial G$ (сюда включается и случай, когда $|\lambda-\xi| \leqslant \varepsilon / 2$, т. е. когда этот круг пуст). Поэтому $\rho\left(\lambda_{k}, \partial G\right) \geqslant|\lambda-\xi|-\varepsilon / 2$. Значит, $\xi$ попадает в кружок $K\left(\lambda_{k}, \rho\left(\lambda_{k}, \partial G\right)+\varepsilon\right)$ и, следовательно, выполнено (iii). Теорема доказана.

5. Следствия. Примеры. Обсуждение результатов. В этом пункте приводятся болеепростые, чем в основном критерии (теореме 2), достаточные, а при дополнительных ограничениях на область $G$ и множество $\Lambda$ и необходимые, условия того, что по системе степеней простейших дробей $F(\Lambda)$ можно представить абсолютно сходящимся в $H_{0}(G)$ рядом произвольную функцию из $H_{0}(G)$. Кроме того, обсуждается взаимосвязь полученных результатов с цитированной во введении теоремой В.П. Хавина и строятся примеры, показывающие сушественность предположений, фигурируюших в теореме 2.

СлЕдСТВИЕ 1. Пусть $G$ конечносвязная область в $\widehat{\mathbb{C}}(G \neq \widehat{\mathbb{C}})$, которая либо является ограниченной в $\mathbb{C}$, либо содержит бесконечно удаленную точку. $E c$ ли множество $M(\Lambda, \partial G)$ плотно в $\partial G$, то выполнено условие (i) теоремы 2 о разлохсении.

ДокаЗАтЕльство. Зафиксируем произвольное $\varepsilon>0$. Так как $M(\Lambda, \partial G)$ плотно в $\partial G$ и $\partial G-$ компакт в $\mathbb{C}$, имеется такая конечная совокупность точек $\left\{z_{k}: k=1, \ldots, m\right\}$ из $M(\Lambda, \partial G)$, что кружки $K\left(z_{k}, \varepsilon\right)$ покрывают $\partial G$. По определению $M(\Lambda, \partial G)$ найдутся $\lambda_{k} \in \Lambda$ с $\rho\left(\lambda_{k}, \partial G\right)=\left|\lambda_{k}-z_{k}\right|, k=1, \ldots, m$ (возможно, разным $z_{k}$ соответствует одна и та же точка из $\Lambda)$. Так как $K\left(\lambda_{k}, \rho\left(\lambda_{k}, \partial G\right)+\varepsilon\right) \supset K\left(z_{k}, \varepsilon\right)$, система $\left\{K\left(\lambda_{k}, \rho\left(\lambda_{k}, \partial G\right)+\varepsilon\right)\right\}_{\lambda_{k} \in \Lambda}$ покрьвает $\partial G$. Следовательно, вьполнено условие (iii) теоремы 2 , в соответствии с которой получаем требуемое. 
СлЕДСТвИЕ 2. Пусть $G-m a$ же, что и в следствии 1, а $\Lambda \subset \partial G$. Тогда условие (i) теоремы 2 әквивалентно плотности $\Lambda$ в $\partial G$.

ДокАЗАТЕЛЬСтво. В рассматриваемом случае $M(\Lambda, \partial G)=\Lambda$. Поэтому достаточность плотности $\Lambda$ в $\partial G$ для (i) вытекает непосредственно из следствия 1 . Аналогично,

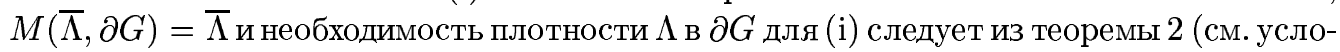
вие (iv)).

ЗАмЕчАния. 1) В случае, когда $G$ представляет собой внешность замкнутого круга произвольного радиуса $R \geqslant 0$ с центром в точке $\lambda \in \mathbb{C}$, следствие 1 - не что иное, как теорема Лорана о разложении любой функции, аналитической вне этого круга и исчезающей в бесконечности, в абсолютно сходящийся в $H_{0}(G)$ ряд вида $\sum_{k=1}^{\infty} a_{k}(z-\lambda)^{-k}$. Ясно, что получить этот результат из теоремы 1 Хавина при $R>0$ не удается, так как в ней требуется, чтобы множество $\Lambda$, образующее систему дробей, было плотньм в круге $\{z:|z-\lambda| \leqslant R\}$.

2 ) Для односвязных областей, когда в качестве континуума выступает $\partial G$, достаточная часть следствия 2 совпадает с утверждением теоремы 1 , а необходимая представляет собой ее обращение.

3) Пусть $\Lambda=\left\{\lambda_{k}, k=1, \ldots, m\right\}$, а область $G$ представляет собой одну из компонент связности внешности в $\widehat{\mathbb{C}}$ объединения замкнутых кругов $\bar{K}\left(\lambda_{k}, r_{k}\right)$, где $r_{k} \geqslant 0$, $k=1, \ldots, m$. Нетрудно видеть, что в данной ситуации $M(\Lambda, \partial G)=\partial G$ и по следствию 1 каждую функцию из $H_{0}(G)$ можно представить в виде суммы ряда $(2)(k$ при этом изменяется в пределах от 1 до $m$ ). Отметим, что этот пример был указан авторам Ю. $\Phi$. Коробейником в связи с одним неточным утверждением, анонсированным в [15]. Именно, в теореме 3 из [15] утверждалось, в частности, что в случае односвязной ограниченной области в $\mathbb{C}$ условие плотности $\Lambda$ в $\partial G(\Lambda$ не обязательно содержится в $\partial G)$ необходимо для того, чтобы система $F(\Lambda)$ была абсолютно представляющей в $H_{0}(G)$. А это, как мы убедились, неверно. Следует сказать, что при доказательстве возможности представления каждой функции из $H_{0}(G)$ абсолютно сходящимся рядом (2) Коробейник исходил из других соображений, существенно учитьвающих специфику рассмотренных в этом примере области $G$ и множества $\Lambda$ и основанных на использовании теоремы Пуанкаре-Ароншайна, которые не удается применить в общей ситуации.

Рассмотрим теперь примеры, показьвающие существенность дополнительных ограничений в теореме 2 для ее справедливости. Прежде чем привести первьй из них, отметим, что из доказательства теоремы 2 следует, что импликация (i) $\Longrightarrow$ (ii) справедлива для произвольных, не обязательно конечносвязных, областей.

ПримеР 1. Пусть $G$ - бесконечносвязная область, полученная из единичного круга $K_{1}=\{z \in \mathbb{C}:|z|<1\}$ удалением точек $z_{n}=1-1 / n(n=1,2, \ldots)$. Рассмотрим произвольное счетноевсюду плотное в $\partial K_{1}$ множество $\Lambda_{1}$ и положим $\Lambda=\Lambda_{1} \cup\left\{z_{n}\right\}_{n=1}^{\infty}$. Нетрудно видеть, что для $G$ и $\Lambda$ вьполнено условия (ii) теоремы 2 . Покажем теперь с помощью теоремы 3 , что $F(\Lambda)$ не является абсолютно представляющей в $H(G)$.

В качестве исчерпьвающей $G$ последовательности областей возьмем $G_{n}=\{z \in \mathbb{C}$ : $\left.|z|<1-(n+1)^{-1}-(n+1)^{-2}\right\} \backslash \bigcup_{k=1}^{n} \bar{K}_{n}\left(z_{k}\right)$, где $\bar{K}_{n}\left(z_{k}\right)=\left\{z \in \mathbb{C}:\left|z-z_{k}\right| \leqslant(n+1)^{-3}\right\}$, $n=1,2, \ldots$.

Рассмотрим последовательность функций

$$
\varphi_{l}(\lambda)=\left\{\begin{array}{l}
1, \text { если } \lambda \in K_{l}\left(z_{k}\right), \quad k \leqslant l, \\
0, \text { если }|\lambda|>1-(l+1)^{-1}-(l+1)^{-2},
\end{array}\right.
$$


$l=1,2, \ldots$. Ясно, что для любого $l \in \mathbb{N}$ функция $\varphi_{l}$ принадлежит $H_{0}\left(G^{c}\right)$ и

$$
\sup _{\lambda_{k} \in \Lambda} \sup _{j \geqslant 1} \frac{\left|\varphi_{l}^{(j-1)}\left(\lambda_{k}\right)\right|}{(j-1) !} \rho^{j}\left(\lambda_{k}, \partial G_{1}\right)<1 .
$$

Тогда если бы для $G$ и $\Lambda$ имело место условие (i) теоремы 2 , то по теореме 3 каждая функция $\varphi_{l}$ обладала бы аналитическим продолжением в некоторое $\bar{G}_{m}^{c}$, непрерьвньгм в $G_{m}^{c}$, где $m$ одно и то же для всех $l \in \mathbb{N}$. Последнее, однако, неверно хотя бы для функции $\varphi_{m+1}$, которая тождественно равна соответственно 1 и 0 в областях $K_{m+1}\left(z_{m+1}\right)$ и $\left\{\lambda:|\lambda|>1-(m+2)^{-1}-(m+2)^{-2}\right\}$, лежащих в одной компоненте связности $\left\{\lambda:|\lambda|>1-(m+1)^{-1}-(m+1)^{-2}\right\}$ множества $\bar{G}_{m}^{c}$.

Итак, мы приходим к выводу, что условие конечной связности $G$ существенно для справедливости импликации (ii) $\Longrightarrow($ i). Отметим, что, практически не меняя проведенных рассуждений, можно доказать, что для рассматриваемой в примере области $G$ нет ни одной системы вида $F(\Lambda)$, являющейся абсолютно представляющей в $H(G)$.

ПримеР 2. Пусть $G$ - полоса $\{z \in \mathbb{C}:|\operatorname{Im} z|<1\}$. В качестве $\Lambda$ возьмем последовательность $\{ \pm i k: k \in \mathbb{N}\}$. Так как при любом натуральном $k$ верно равенство $\rho( \pm i k, \partial G)$ $=k-1$ и при любом $\varepsilon>0$

$$
\bigcup_{k \in \mathbb{N}} K( \pm i k, k-1+\varepsilon)=\{z \in \mathbb{C}:|\operatorname{Im} z|>1-\varepsilon\}
$$

для $G$ и $\Lambda$ вьполнено условие (iii) теоремы 2. С другой стороны, условие (ii), а следовательно, и (i) этой теоремы места не имеют. В самом деле, достаточно в качестве $S$ взять, например, отрезок мнимой оси $\{i y:|y| \leqslant 1 / 2\}$. Тогда $\bigcup_{k \in \mathbb{N}} K( \pm i k, S)=\{z \in \mathbb{C}$ : $|\operatorname{Im} z|>1 / 2\}$ и условие (ii) нарушается. Таким образом, требование ограниченности $\partial G$ существенно для справедливости импликации (iii) $\Longrightarrow$ (i).

ПРИмеР 3. Пусть $G$ - квадрат $\{z \in \mathbb{C}:|\operatorname{Re} z|<1,|\operatorname{Im} z|<1\}$. Расположим $\lambda_{k}$ на осях координат: $\Lambda:=\{ \pm k, \pm i k \mid k \in \mathbb{N}\}$. Легко видеть, что $\bar{\Lambda}=\Lambda, M(\bar{\Lambda}, \partial G)$ состоит всего из четырех точек $\pm 1, \pm i$ и, значит, условие (iv) теоремы 2 не вьполняется. Тем не менее, ясно, что (iii), а тогда и (i) в данном случае выполнены. То есть, дополнительное требование ограниченности $\Lambda$ в теореме 2 необходимо для эквивалентности (i) и (iv).

В заключение остановимся коротко на вопросах существования абсолютно представляющих в $H_{0}(G)$ систем вида $F(\Lambda)$ и единственности представлений вида $(2)$ для элементов из $H_{0}(G)$.

В соответствии со следствием 2 для любой конечносвязной области $G \neq \widehat{\mathbb{C}}$, либо являющейся ограниченной в $\mathbb{C}$, либо содержащей бесконечно удаленную точку, всегда имеется система $F(\Lambda)$, по которой можно представить в виде абсолютно сходящегося в $H_{0}(G)$ ряда $(2)$ произвольную функцию из $H_{0}(G)$. Для этого достаточно взять всюду плотное в $\partial G$ не более чем счетное множество $\Lambda$. Однако если $G$ не содержит бесконечно удаленную точку, но является неограниченной в $\mathbb{C}$, то для нее уже может и не существовать абсолютно представляющей в $H(G)$ (в данном случае $H_{0}(G)=H(G)$ ) системы $F(\Lambda)$. Например, допустим, что область $G$ в $\mathbb{C}$, отличная от всей плоскости, содержит полуплоскость. Для определенности пусть $\{z \in \mathbb{C}: \operatorname{Re} z>0\} \subset G$. Тогда для компакта $\Pi=\{z \in \mathbb{C}|1 \leqslant \operatorname{Re} z \leqslant 2,| \operatorname{Im} z \mid \leqslant 1\}$ в $G \rho(\lambda, \partial \Pi)=\rho(\lambda, I)$, где 
$I=\{z \in \mathbb{C}|\operatorname{Re} z=1,| \operatorname{Im} z \mid \leqslant 1\}, \operatorname{Re} \lambda \leqslant 0$. Поэтому ни одна точка вещественной оси, лежащая правее $z=1$, не покрывается системой кругов $\{K(\lambda, \rho(\lambda, \partial \Pi))\}$, когда $\lambda$ пробегает полуплоскость $\operatorname{Re} \lambda \leqslant 0$ и, тем более, когда $\lambda \in \mathbb{C} \backslash G$. Это означает, что для всего $\mathbb{C} \backslash G$ не выполнено условие (ii) теоремы 2 , а следовательно, ни для одного не более чем счетного множества из $\mathbb{C} \backslash G$ не имеет места и условие (i) этой теоремы.

Единственность представления (2) произвольной функции из $H_{0}(G)$ существенно зависит от области $G$ и структуры $\Lambda$. Например, если $G$ - внешность некоторого круга, а $\Lambda$ состоит лиш из одной точки - центра этого круга, то по теореме Лорана имеем единственность подобных представлений. С другой стороны, если, к примеру, $G$ - внешность объединения двух кругов: $\{z \in \mathbb{C}:|z| \leqslant 1\}$ и $\{z \in \mathbb{C}:|z-1| \leqslant 1\}$, то по следствию 1 система $\left\{z^{-j},(z-1)^{-j}\right\}_{j \in \mathbb{N}}$ является абсолютно представляющей в $H_{0}(G)$. При этом функция $f(z)=1 / z$ имеет, по крайней мере, два различных представления по этой системе: $1 / z$ и $\sum_{j=1}^{\infty}(-1)^{j-1}(z-1)^{-j}$, абсолютно сходящихся в $H_{0}(G)$. Другие примеры подобной неединственности хорошо известны и приведены, например, в [5]-[7].

\section{СПИСОК ЦИТИРОВАННОЙ ЛИТЕРАТУРЫ}

[1] Wolff J. Sur les séries $\sum_{k=1}^{\infty} A_{k} /\left(z-\alpha_{k}\right) / /$ C. R. Acad. Sci. 1921. V. 173. P. 1327-1328.

[2] Denjoy A. Sur les séries de fractions rationnelles // Bull. Soc. Math. France. 1924. V. 52. P. 418-434.

[3] Гончар А. А. О квазианалитическом продолжении аналитических функций через жорданову дугу // Докл. АН СССР. 1966. Т. 166. № 5. С. 1028-1031.

[4] Леонтьева Т. А. Представление функций, аналитических в замкнутой области, рядами рациональных функций // Матем. заметки. 1968. Т. 4. № 2. С. 191-200.

[5] Коробейник Ю.Ф. К вопросу о разложении аналитических функций в ряды по рациональным функциям // Матем. заметки. 1982. Т. 31. № 5. С. 723-737.

[6] Сибилев Р. В. Теорема единственности для рядов Вольфа-Данжуа // Алгебра и анализ. 1995. Т. 7. №1. С. 170-199.

[7] Хавин В.П. Об одной задаче В.В.Голубева // Докл. АН СССР. 1959. Т. 126. №3. C. 511-513.

[8] Хавин В.П. Один аналог ряда Лорана // Исследования по совр. пробл. теории функций компл. переменного. М.: ГИФМЛ, 1961. С. 121-131.

[9] Канторович Л. В., Акилов Г. П. Функциональный анализ в нормированных пространствах. М: Физматгиз, 1959.

[10] Коробейник Ю.Ф. Представляющие системы // УМН. 1981. Т. 36. № 1. С. 73-126.

[11] Köthe G. Dualität in der Funktionentheorie // J. Reine Angew. Math. 1953. V. 191. P. 30-49.

[12] Хавин В.П. Пространства аналитических функций // Итоги науки. Матем. анализ. М.: Инст. научн. информ, 1966. С. 76-164.

[13] Эдвардс Р. Функциональный анализ. М.: Мир, 1969.

[14] Уолш Дж. Л. Интерполяция и аппроксимация рациональными функциями в комплексной области. М.: ИЛ, 1961.

[15] Семенова Г. А. К вопросу о представлении аналитических функций рядами рациональных функций // Совр. методы в теории краевых задач. Тезисы докл. школы. Воронеж: ВГУ, 1997. C. 136 . 